as by examining the Reggio Emilia district database for rare diseases. To be included in the study, patients must satisfy the following 2 criteria: Age at disease onset $\geq 50$ years; evidence of large-vessel vasculitis by clinical criteria, angiography, MRA, CTA, PET/CT and/or ultrasonography. Demographic, clinical, laboratory and imaging data collected at first visit were retrived from patients records. Results: There were 93 incident cases of LV GCA (66 women, 71\%) during the 12 year study period; Mean \pm SD age at diagnosis was $72 \pm 9$ years. The three most prevalent signs an symptoms were: systemic in 49 pts $(52.7 \%)$, GCA cranial symptoms in 39 pts (41.9\%) and PMR in 35 pts (37.6\%). Peripheral ischaemic symptoms were observed only in 8 pts $(8.6 \%)$. Forty four pts had temporal artery biopsy and $70.5 \%$ resulted positive. At US examination the three most common involved arteries were: common carotid artery (59.2\% pts), subclavian artery (53.3\% pts) and axillary artery (49.3 pts). At PET/CT scan examination the most common involved arteries were: thoracic aorta $(72.1 \% \mathrm{pts})$, subclavian artery $(61.8 \% \mathrm{pts})$ and abdominal aorta (58.8\% pts). Celiac trunk and mesenteric artery were involved in $18.4 \%$ of pts and renal artery in $10.2 \%$ of pts.

Conclusions: In an inception cohort of LVGCA systemic manifestations had the highest prevalence among presenting symptoms. Imaging studies demonstrated an high prevalence of aortic and subclavian involvement.

Disclosure of Interest: None declared

DOI: 10.1136/annrheumdis-2018-eular.5263

\section{AB0694 GIANT CELL ARTERITIS EPIDEMIOLOGY IN LA REUNION: A RETROSPECTIVE CASES SERIES}

Q. Richier ${ }^{1}$, T. Deltombe ${ }^{1}$, A. Foucher ${ }^{2}$, C. Roussin ${ }^{3}$, A. Randrianjohany ${ }^{4}$, A. Gerber ${ }^{1}$, P. Poubeau" ${ }^{2}$, L. Raffray ${ }^{1} .{ }^{1}$ Internal Medicine, CHU de La Réunion, Saint Denis; ${ }^{2}$ Internal Medicine, $\mathrm{CHU}$ de La Réunion, Saint Pierre; ${ }^{3}$ Internal Medicine, CH Gabriel Martin, Saint Paul; ${ }^{4}$ Internal Medicine, Groupe Hospitalier Est Réunion, Saint Benoît, France

Background: Giant Cell Arteritis (GCA) is the most common vasculitis in people over 50 years, with incidence varying according to geographic location. In Europe, the average incidence is $10-30$ per 100000 inhabitants over 50 years, whereas in Africa and Asia it is approximately 1.5 per 100000 .

Objectives: To define GCA epidemiology in La Réunion, a French oversea territory in South West Indian Ocean characterised by several ethnic groups and genetic admixture.

Methods: Retrospective study between January 2005 and August 2017 in the 4 main hospitals of La Réunion. Patients were identified through hospital informatics databases and practitioner records. A definite diagnosis of GCA was considered for patients fulfilling ACR criteria and/or by assessment by a trained rheumatologist. Incidence and prevalence calculation was based on French national census data.

Results: Sixty patients were included, of which $60 \%$ were women. Mean age at diagnosis was 73.7 years, with a mean delay to diagnosis of 6.8 months. Cases met an average of $3.78 / 5$ ACR criteria, and $78 \%$ had $>2$ criteria. The mean annual incidence was 2.33 per 100000 inhabitants older than 50 years $(203,000)$, with $95 \%$ confidency interval (IC) of 1.74-2.92. The prevalence at the end of period study was 24 cases per 100,00 (IC: 18-30). There was no seasonal variation regarding disease onset. Clinically, patients complained of asthenia and headache in $75 \%$, fever in $33 \%$ and ophthalmologic damage for $32 \%$ of the cases, of which 5 had anterior ischaemic optic neuropathy. Polymyalgia rheumatica was associated in $42 \%$ of all cases. Total blood cells counts were usually within normal values, whereas mean CRP was $111 \mathrm{mg} / \mathrm{L}$. Anicteric cholestasis was a common finding: mean GGT=78 IU/L $(n<42)$ and alkaline phosphatase $=107 \mathrm{IU} / \mathrm{L}(\mathrm{n}<104)$ Radiological examinations contributed to diagnosis in only $9 / 31$ cases. Temporal artery biopsy was performed in $91 \%$ of patients and showed specific histological features of GCA in 55\%. Corticosteroid regimen was introduced in 59 patients (1 died the day after the biopsy) and mean treatment duration was 26 months, for a total dose of $10.6 \mathrm{~g}$. Antiplatelet therapy was given in $47 \%$ of patients. One patient in three experienced one or more relapses.

Conclusions: This is the first study to describe GCA in La Réunion, and more generally in Indian Ocean. It shows an incidence 4-12 times lower than in most European countries with white ancestry background. This discrepancy could be explained by the contribution of the various ethnic groups of La Reunion, especially those coming from parts of the world characterised by a lower GCA incidence (Africa, India, South East Asia). A shorter life expectancy may also account for this observation, assuming that GCA incidence increases with age. Sex ratio and age at diagnosis were similar to European studies, as well as clinico-biological features, response to treatment and side effects. Some limitations of our study should be taken in consideration: inclusions of hospitalised patients only, informatics record limits and retrospective design that did not afford for ethnic background determination.

\section{REFERENCE:}

[1] Mahr A, et al. [Epidemiology and natural history of giant cell arteritis]. Rev Med Interne. 2017 Oct;38(10):663-669.

Disclosure of Interest: None declared DOI: 10.1136/annrheumdis-2018-eular.5814

\section{AB0695 2 PREDICTIVE FACTORS OF LONG-TERM CLINICAL OUTCOME IN BEHCET'S SYNDROME PATIENTS WITH OCULAR INVOLVEMENT: DOES ACTUALLY THE DISEASE TEND TO GROW DIM OVER TIME?}

R. Talarico ${ }^{1}$, M. Figus ${ }^{2}$, C. Posarelli ${ }^{2}$, A. d'Ascanio ${ }^{1}$, R. Neri ${ }^{1}$, C. Tani ${ }^{1}$, C. Baldini ${ }^{1}$, M. Mosca ${ }^{1}{ }^{1}$ Department of Clinical and Experimental Medicine, University of Pisa, Rheumatology Unit, ${ }^{2}$ Department of Surgical, Medical, Molecular Pathology and Emergency, University of Pisa, Pisa, Italy, Ophthalmology Unit, Pisa, Italy

Background: Behçet's syndrome (BS) is a multisystemic, chronic relapsing inflammatory disease classified among the vasculitis. Eye involvement represent one of the most serious manifestation of BS and occurs in half of all patients. It seems more frequent and severe among young males and, unluckily, it still represents a significant cause of morbidity.

Objectives: The aims of the study were: to study the relapse rate of BS patients with ocular involvement and to identify factors able to predict long-term outcome Methods: Among a cohort of 138 patients with a diagnosis of BS according the ISG criteria, 62 patients ( 37 males and 25 females; mean age at the onset $38 \pm 5$ years) with ocular involvement were prospectively studied. The probability of clinical relapse after remission of the first ocular attack was calculated using the Kaplan-Meier method. Predictors of long-term outcome were identified by univariate analysis using the log-rank test and by multivariate analysis using Cox proportional hazards regression models.

Results: The mean time between the first initial symptoms of BS and the onset of eye lesions was $2 \pm 2$ years. The number of ocular attacks were the following: 43 posterior uveitis, 27 anterior uveitis, 26 retinal vasculitis, while panuveitis developed in 18 subjects. The cumulative relapse rates at 1 year, 3 years, and 5 years after remission of the first ocular attack were $41 \%, 31 \%$, and $28 \%$, respectively. On multivariate analysis, a younger age ( $<30$ years) at the onset of ocular involvement, male sex and medical treatments other than biological agents represent independent predictive factors for more early relapses in BS patients with ocular involvement.

Conclusions: The relapse rate seems to be more frequent in the first years of diseases, and probably it could be related to the fact the disease tend to grow dim over time. As literature data suggest, younger age and male sex still represent predictive factors of poor long-term clinical outcome.

Disclosure of Interest: None declared

DOI: 10.1136/annrheumdis-2018-eular.7039

\section{AB0696 CLINICAL CHARACTERISTICS AND OUTCOME OF PATIENTS WITH GRANULOMATOSIS WITH POLYANGIITIS (GPA) ACCORDING TO ANCA POSITIVITY AND SPECIFICITY}

R. Solans-Laque ${ }^{1}$, J. Rios ${ }^{2}$, M. Rodriguez-Carballeira ${ }^{3}$, B. Frutos ${ }^{4}$, L. Saez ${ }^{5}$, F. Pasquau ${ }^{6}$, J. Oristrell ${ }^{7}$, E. Fonseca ${ }^{8}$, G. Fraile ${ }^{9}$, on behalf of REVAS-GEAS (SEMI). 'Autoimmune Diseases Unit. Internal Medicine Department, Vall-Hebron Hospital, Barcelona; ${ }^{2}$ Internal Medcine, $\mathrm{H}$ La-Paz, Madrid; ${ }^{3}$ Internal Medicine, $\mathrm{H}$ Mutua, Terrassa; ${ }^{4}$ Internal Medcine, H Fuenlabrada, Madrid; ${ }^{5}$ Internal Medcine, $H$ Miguel-Servet, Zaragoza; ${ }^{6}$ Internal Medcine, H Marina-Baixa, Alicante; ${ }^{7}$ Internal Medicine, H Parc-Tauli, Sabadell; ${ }^{8}$ Internal Medcine, H Cabueñes, Asturias; ${ }^{9}$ I Medicine, H Ramon-Cajal, Madrid, Spain

Background: GPA is a necrotizing granulomatous vasculitis associated with ANCA that usually involves ENT, lung and kidneys. ANCA are mostly directed against proteinase 3 (PR3), although in few cases are directed against myeloperoxidase (MPO) or negative.

Objectives: To analyse the phenotype, clinical course and outcome of patients with GPA according to ANCA positivity and specificity

Methods: multicenter retrospective-longitudinal study that included patients diag nosed with AAV between Jan 1995 and Jan 2016 in 21 Hospitals from Spain (REVAS-Study). We analysed the clinical characteristics, treatment and outcome depending on the ANCA positivity and specificity. Statistical analysis was performed using SPSSvs. 20

Results: 221 patients with GPA were included:162 with PR3-ANCA, 36 with MPO-ANCA, and 23 with negative-ANCA. The mean-age at disease onset was higher in patients with MPO-ANCA (59.9ロ16.5 years) than in patients with PR3

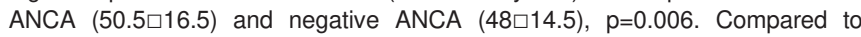

